\title{
Superparamagnetic nickel colloidal nanocrystal clusters with antibacterial activity and bacteria binding ability
}

\author{
Bo Peng ${ }^{1 * *}$, Xinglin Zhang ${ }^{2,3^{\dagger}}$, Dirk G.A.L. Aarts ${ }^{1}$ and Roel P.A. Dullens ${ }^{1 *}$ \\ ${ }^{1}$ Department of Chemistry, Physical and Theoretical Chemistry Laboratory, University of \\ Oxford, Oxford, South Parks Road, Oxford OX1 3QZ, UK; ${ }^{2}$ College of Biosystem \\ Engineering and Food Science, Zhejiang University, Hangzhou 310058, China; ${ }^{3}$ Sir William \\ Dunn School of Pathology, University of Oxford, South Parks Road, Oxford OX1 3RE, UK. \\ ${ }^{\dagger}$ These authors contributed equally to this work. \\ *e-mail: pengbo006@gmail.com; roel.dullens@chem.ox.ac.uk
}

\begin{abstract}
Recent progresses in synthetic nanotechnology and the ancient use of metals in food preservation and antibacterial treatment of wounds, have prompted the development of nanometallic materials for antimicrobial applications ${ }^{1-4}$. The materials designed so far however do not simultaneously display antimicrobial activity and the capability of binding and capturing bacteria and spores. Here, we develop a one-step pyrolysis procedure to synthesise monodisperse superparamagnetic nickel colloidal nanocrystal clusters (SNCNC), which show both antibacterial activity and the ability of binding Gram-positive (Bacillus subtilis) and Gramnegative (Escherichia coli) bacteria, as well as bacterial spores. The SNCNC are formed by rapid burst of nickel nanoparticles, which then slowly self-assemble into clusters. The clusters can magnetically extract $\mathbf{9 9 . 9 9 \%}$ of bacteria and spores and provide a promising approach for the removal of microbes, including hard-to-treat microorganisms. We believe that our work illustrates the exciting opportunities that nanotechnology offers for alternative antimicrobial strategies and other applications in microbiology.
\end{abstract}

The antibacterial nature of metals was noticed and utilised long before the first observation of bacteria by Antonie van Leeuwenhoek in $1676^{1}$. For instance, the Phoenicians stored water, wine and vinegar in silver-coated containers to limit bacteria contamination ${ }^{2}$; and copper was recommended to sterilise chest wounds against bacterial infection in ancient Egypt ${ }^{3}$. With the identification and mass production of antibiotics in the 1930s, which led to a dramatic decline in mortality rates caused by bacterial infections, the antibacterial use of metals decreased. However, regularly occurring outbreaks of infectious diseases caused by antibiotic resistant bacteria gradually tempered the use of antibiotics. Bacteria were found to be intrinsically resistant or have acquired resistance to any one or multiple (superbugs) of the available antibiotics. As such, bacterial infections are on the rise and bacteria are likely to develop further resistance to new antibiotics, which calls for the development of alternative antimicrobial strategies 5 .

Recently, nanometallic materials regained significant attention as antimicrobial systems thanks to the unique properties that arise at the nanoscale ${ }^{6-9}$. Nanoparticles exhibit superior antibacterial activity against a wide range of bacteria compared to their counterpart bulk materials ${ }^{6,10}$. In addition, when the magnetic dipoles in ferro- or ferri-magnets are confined to nanoscale dimensions, these materials behave superparamagnetically at room temperature ${ }^{11}$. Based on these inherent features and the 
potential to functionalise their surfaces, nanometallic materials are promising systems from both a fundamental and a practical antimicrobial perspective $\mathrm{e}^{8,12}$.

One particular class of nanometallic materials, iron-based systems, are widely used as nanocarriers for drug delivery because of their magnetic recyclability and bacterial benignancy ${ }^{8,12,13}$. To be suitable as nanocarriers, their surface is functionalised with antibodies, proteins, dyes and other nanocomponents, which mediate the interactions between bacteria and nanoparticles ${ }^{14-17}$. In this way, bacteria can be magnetically separated regardless of their viability ${ }^{18-22}$. However, functional entities that bridge magnetic-nanocarriers and bacteria are challenging to prepare ${ }^{18-22}$. Introducing a magnetic material alternative to iron-based systems may circumvent the problems encountered during the selection and preparation of these functional entities, and may introduce novel properties potentially useful for antimicrobial applications ${ }^{14-16}$.

Here, we report a simple wet synthesis of superparamagnetic nickel colloidal nanocrystal clusters (SNCNC) with inherent antibacterial and bacterial-binding characteristics. We find that nickel nanocrystals spontaneously self-organise into SNCNC with tunable sizes and magnetic moment. In addition, the binding pattern of SNCNC to bacteria is species dependent. The combined binding and antibacterial activities of SNCNC allow over $99.99 \%$ of both Gram-positive (Bacillus subtilis) and Gram-negative (Escherichia coli) bacteria to be captured, killed and magnetically removed. Importantly, the different time scales involved in binding and killing may enable the extraction and manipulation of bacteria. Furthermore, the binding is also applicable to (B. subtilis) spores, providing a valuable route for the removal of these hard-to-treat bugs.

The SNCNC are synthesised through pyrolysis of $\mathrm{NiCl}_{2}$ to zero-valent-stated $\mathrm{Ni}$ aided by $\mathrm{NaOH}$ and stabilised by trisodium citrate ( $\mathrm{NaCit}$ ) in a reductive diethylene glycol (DEG) environment. DEG functions both as a polar solvent with a high boiling point, facilitating an easy adjustment of the reaction kinetics and the hydrophilicity of the products, and as a reductant. The hot injection of $\mathrm{NaOH} / \mathrm{DEG}$ into the mixture of $\mathrm{NiCl}_{2}, \mathrm{DEG}$ and $\mathrm{NaCit}$ changes its colour from transparent to opaque green, to brown, and ultimately to black over a brief interval of time $(<2 \mathrm{~min})$, indicating a rapid precipitation, dehydration and reduction to Ni (see Supplementary information: SI, and supplementary figure-1: SF-1). Monitoring the reaction with transmission electron microscopy reveals a timedependence of the sample morphology. As illustrated in Figure 1, the reaction experiences a burst nucleation and nuclei grow to primary nanoparticles over a fairly short period of time $(<2 \mathrm{~min})$. Thereafter, a relatively slow yet gradual clustering occurs $(\sim 1 \mathrm{~h})$. The results from transmission electron microscopy, energy dispersive X-ray spectroscopy, X-ray diffraction, and X-ray photoelectron spectroscopy (XPS) complementarily verify the formation of Ni and the structure of SNCNC as demonstrated in Figure 1e-g and SF-2. The SNCNC comprise a large number of Ni nanograins $(<10 \mathrm{~nm})$ with anisotropic crystalline orientations (see the insets in Figure 1e) and behave superparamagnetic at $300 \mathrm{~K}$ and ferromagnetic at $5 \mathrm{~K}$ as demonstrated in Figure $1 \mathrm{~h}$. Confining the individual magnetic dipoles to such an extent that the thermal energy becomes sufficiently high to randomise the magnetic dipoles defines the superparamagnetic regime ${ }^{11,23}$. In contrast, lowering the temperature to a level which is insufficient to perturb the alignment of magnetic dipole brings the material back to the ferro- or ferri-magnetic state. The transition point (blocking temperature) of SNCNC is measured to be $260 \mathrm{~K}$ (see the inset in Figure 1h).

Conveniently, we can easily tune the morphology and magnetic properties of SNCNC by altering the concentration of the reactants added and the reaction temperature. The average size of the SNCNC can be tuned in the range of 100-300 nm by controlling the molar ratio of $\mathrm{NaOH}$ and $\mathrm{NiCl}_{2}$ or the amount of NaCit, while the size distribution of SNCNC remains narrow (see Figure 2a-e and SF-3 and 
4). More specifically, at molar ratios of $\mathrm{NaOH} / \mathrm{NiCl}_{2}$ over 2 (SF-3) and concentrations of $\mathrm{NaCit}$ over $10 \mathrm{mg} / \mathrm{mL}$ (SF-4) monodisperse SNCNC are obtained, while beyond that polydisperse samples are found (SF-3 and 4). Increasing the $\mathrm{NaOH} / \mathrm{NiCl}_{2}$ ratio or decreasing the $\mathrm{NaCit}$ concentration, leads to a decrease in size of the SNCNC. Note that the cluster morphology is observed even in the absence of $\mathrm{NaCit}$ (SF-4a), implying that NaCit does not affect the primary nanoparticle assembly into clusters. In addition, the solvent (DEG) allows a wide range of reaction temperatures to be used, which affects the magnetic properties of the SNCNC. A stepwise drop in reaction temperature from 240 to $160{ }^{\circ} \mathrm{C}$ decreases the mass magnetic moment $(M)$ from 31 to $15 \mathrm{emu} / \mathrm{g}$, whereas the superparamagnetic properties are conserved (see Figure $2 \mathrm{f}$ and $\mathrm{g}$ ). Similar to the magnetisation behaviour observed in bulk (Figure 2f), the mean magnetisation per grain, $\mu$, exhibits an increase as a function of the reaction temperature (see Figure 2g; for detailed calculation see SI: SF-5 and supplementary table 1, ST-1). The magnetic moment of the clusters will obviously be much higher than $\mu$, which renders them much more responsive to external magnetic fields ${ }^{24}$. Further lowering the reaction temperature to $150{ }^{\circ} \mathrm{C}$ leads to a non-magnetic sample, consistent with a critical temperature to induce the reduction ${ }^{25}$.

The obtained SNCNC are stable in polar environments. To elucidate the origin of this hydrophilicity, XPS was performed to characterise the surface properties of the SNCNC. Besides Ni, XPS also detects the presence of carbon (SF-2), which originates from NaCit and DEG. Independent Fouriertransform infrared spectroscopy (SF-6) corroborates this finding. Indeed, thermo-gravimetric analysis (SF-7) shows that a small amount $(\leq 7 \mathrm{wt} \%)$ of NaCit and DEG is built into the SNCNC, consistent with the hydrophilic nature of the SNCNC. As a consequence, our SNCNC are stable in aqueous environments relevant for biological systems, such as saline and phosphate-buffered saline media, unlike most nickel nanoparticles which are typically stable in apolar environments ${ }^{12}$.

Despite the fact that Ni has been well-recognised as an essential micronutrient required by organisms, the exposure to a Ni-rich environment might be poisonous or lethal to organisms ${ }^{26}$. Coupling this toxicity with superparamagnetism potentially allows for the use of SNCNC as a recyclable antibacterial system. Dispersing SNCNC into bacterial suspensions, surprisingly, shows that they can bind and thereby capture both Gram-positive B. subtilis and Gram-negative E. coli bacteria, as displayed in Figure 3a and d. In contrast, superparamagnetic $\mathrm{Fe}_{3} \mathrm{O}_{4}$ colloidal nanocrystals (SFCN, for synthesis detail see SI, section 1.2.4) do not show the ability to capture bacteria, as is evident from the supernatants being as opaque as the dispersions with bacteria only. The SFCN control experiment thus suggests that the bacteria-binding ability of SNCNC may be ascribed to the presence of nickel. The sub-micron size of the SNCNC allows for the direct real space and time observation of the bacteria binding to SNCNC and, interestingly, a microscopic inspection of the sediment (see Figure $3 \mathrm{~b}$ and e) reveals different binding patterns of SNCNC to B. subtilis and E. coli bacteria. While SNCNC homogeneously cover the surface of $B$. subtilis, they favour attaching to the cell poles of E. coli (see the insets in Figure $3 \mathrm{~b}$ and e, and SF-12). Additionally, the disk diffusion assay (Figure $3 \mathrm{c}$ and $\mathrm{f}$ ) clearly shows the antibacterial nature of our Ni-clusters against both B. subtilis and E. coli, in contrast to that of SFCN, which shows no antibacterial activity. We note that nickel nanoparticles may have been a more appropriate control for the antibacterial experiments, however, these particles are typically stable in apolar solvents ${ }^{12}$ rendering them unsuitable as controls in aqueous bacteria suspensions.

Next, we discuss as to why the SNCNC may bind differently to the two bacteria considered here. While this process is probably very complex and not fully understood, the surface properties of the Gram-positive B. subtilis and Gram-negative E. coli bacteria are likely to play a key role. To this end we fully characterised the properties of the SNCNC (SI, section 2.1), the DEG stabilised SFCN control and two further controls: SNCNC stabilised by DEG only, i.e. without NaCit (for synthesis 
detail see SI, section 1.2.2) and $\mathrm{NiO}$ particles synthesised from the SNCNC by rising the temperature to $300{ }^{\circ} \mathrm{C}$ (SI, section 1.2.3). The characterisation of the controls are summarised in section 2.2 of the SI. First of all, we find that both the bacteria and the SNCNC - with and without NaCit - are all negatively charged (ST-2), which rules out electrostatics as the main origin for bacteria binding. Also, the bacteria are not simply binding to $\mathrm{NaCit}$ as both $\mathrm{SNCNC}$ with and without $\mathrm{NaCit}$ can bind to bacteria (Figure 3, SF-12, and SF-13). This is corroborated by the fact that SNCNC stabilised by DEG only can also bind to bacteria (SF-13). Furthermore, we observe that negatively charged SFCN (ST-2) stabilised by DEG only (SF-10) do not show any binding to the bacteria (Figure 3a and d, SF-14, SF15, and ST-3) and neither do the NiO particles (SF-16). Hence, the common factor for the bacteria binding activity is the stable DEG-grafted SNCNC (ST-4).

The difference in binding of the SNCNC to B. subtilis and E. coli - as observed in Figure $3 \mathrm{~b}$ and e, SF-12, and SF-13 - suggests that SNCNC may interact with particular components at the bacterial surface. To check the affinity of the SNCNC for different chemical groups, they were transferred from an aqueous medium to an apolar medium (chloroform) in the presence of different chemicals with the same apolar group $\left(-\mathrm{C}_{17} \mathrm{H}_{33}\right)$ but different polar functional groups: $-\mathrm{NH}_{2}$ (oleylamine), - $\mathrm{COOH}$ (oleic acid) and - $\mathrm{OH}$ (oleyl alcohol). The affinity between these different chemicals and the SNCNC is then reflected by the minimum amount required to successfully disperse the SNCNC in chloroform (see SI, section 1.2.6). As shown SF-17, we found the largest affinity for oleylamine $\left(-\mathrm{NH}_{2}\right)$, then oleyl alcohol $(-\mathrm{OH})$ and finally oleic acid $(-\mathrm{COOH})$. This suggests that the bacteria binding activity of the SNCNC is mainly due to the binding between SNCNC and the amino groups on the surface of the bacteria. This seems consistent with the observation that SNCNC homogeneously cover the surface of B. subtilis, which has an outer layer of peptidoglycan, a polymer consisting of sugars and amino acids. Similarly, the preferential bonding of SNCNC at the cell poles of E. coli, which has an outer layer of lipopolysaccharides (LPS), large molecules consisting of lipids and polysaccharides ${ }^{27}$, may be ascribed to the proteins - rich in amino groups - that are exposed at the E. Coli cell poles ${ }^{28,29}$.

Finally, we quantitatively analyse the binding and antibacterial activity of SNCNC to B. subtilis and $E$. coli. As shown in Figure 4a and b, the overall viability of both bacteria diminishes with time and with increasing SNCNC concentration, although $B$. subtilis bacteria are significantly more vulnerable to SNCNC than E. coli. While the planktonic viabilities of both bacteria show the same trend as the overall viability, that of $B$. subtilis again drops faster, suggesting a more efficient binding of $B$. subtilis by SNCNC. The difference in vulnerability and capturing may be associated with the different binding behaviour of SNCNC to both bacteria (see Figure $3 \mathrm{~b}$ and e). Interestingly, the different time scales involved in the antibacterial and capturing assays imply that there is a time window to collect and extract live bacteria using SNCNC. In fact, SNCNC can be recycled until their surfaces are saturated. As illustrated in Figure 4c, $0.5 \mathrm{mg}$ of the same SNCNC is sufficient to successfully remove $10^{6}$ bacteria in three consecutive cycles (see SI, section 1.2.9), before the removal efficiency drops significantly. Importantly, we also applied the capturing ability of SNCNC to B. subtilis spores (Figure 4d), which are extremely resistant to heat, radiation and all routinely used antimicrobial agents $^{30}$. While the antisepsis activity is minor, $99.99 \%$ of the spores can be removed within 40 min by SNCNC, which may serve as a new approach for clearing these hard-to-treat bugs.

In conclusion, we have demonstrated a single-step synthesis of superparamagnetic nickel colloidal nanocrystal clusters (SNCNC) that exhibit antibacterial and capturing abilities to both Gram-positive and Gram-negative bacteria, as well as bacterial spores. The formation of SNCNC in polar media at high temperatures involves the formation of nickel nanoparticles, which subsequently self-assemble into clusters. The magnetic properties and morphology of the SNCNC can be easily tuned by varying the reaction temperature and the concentration of the reactants, respectively. Furthermore, we 
quantitatively characterised the antibacterial activity of the SNCNC, and identified the capturing ability of SNCNC to bacteria and spores. This work provides a promising route for the removal or extraction of bacteria and bacterial spores, and represents an example of fruitfully integrating nanotechnology and antimicrobial strategies.

\section{References}

1. Porter, J. R. Antony van Leeuwenhoekl: Tercentenary of his discovery. Bacteriol. Rev. 40, 260-269 (1976).

2. Alexander, J. W. History of the medical use of silver. Surg. Infect. 10, 289-292 (2009).

3. Dollwet, H. H. A. \& Sorenson, J. R. J. Historic use of copper-compounds in medicine. Trace Elem. Med. 2, 80-87 (1985).

4. $\quad$ Langer, R. \& Tirrell, D. A. Designing materials for biology and medicine. Nature 428, 487492 (2004).

5. Schaberle, T. F. \& Hack, I. M. Overcoming the current deadlock in antibiotic research. Trends Microbiol. 22, 165-167 (2014).

6. Sharma, V. K., Yngard, R. A. \& Lin, Y. Silver nanoparticles: Green synthesis and their antimicrobial activities. Adv. Colloid Interface Sci. 145, 83-96 (2009).

7. Seil, J. T. \& Webster, T. J. Antimicrobial applications of nanotechnology: methods and literature. Int. J. Nanomedicine 7, 2767-2781 (2012).

8. Pankhurst, Q. A., Connolly, J., Jones, S. K. \& Dobson, J. Applications of magnetic nanoparticles in biomedicine. J. Phys. D-Applied Phys. 36, R167-R181 (2003).

9. Richter, A. P. et al. An envrionmentally benign antimicrobial nanoparticle based on a silverinfused lignin core. Nat. Nanotechnol. 10, 817-823 (2015).

10. Chernousova, S. \& Epple, M. Silver as antibacterial agent: Ion, nanoparticle, and metal. Angew. Chemie - Int. Ed. 52, 1636-1653 (2013).

11. Frenkel, J. \& Doefman, J. Spontaneous and induced magnetisation in ferromagnetic bodies. Nature 126, 274-275 (1930).

12. Lu, A. H., Salabas, E. L. \& Schüth, F. Magnetic nanoparticles: Synthesis, protection, functionalization, and application. Angew. Chemie - Int. Ed. 46, 1222-1244 (2007).

13. Weissleder, R. et al. Superparamagnetic iron oxide: Pharmacokinetics and toxicity. Am. J. Roentgenol. 152, 167-173 (1989).

14. Vikesland, P. J. \& Wigginton, K. R. Nanomaterial enabled biosensors for pathogen monitoring - a review. Environ. Sci. Technol. 44, 3656-3669 (2010).

15. Miller, K. P., Wang, L., Benicewicz, B. C. \& Decho, A. W. Inorganic nanoparticles engineered to attack bacteria. Chem. Soc. Rev. 44, 7787-7807 (2015).

16. Gao, J., Gu, H. \& Xu, B. Multifunctional magnetic nanoparticles: design, synthesis, and biomedical applications. Acc. Chem. Res. 42, 1097-1107 (2009).

17. Taylor, E. N. et al. Superparamagnetic iron oxide nanoparticles (SPION) for the treatment of 
antibiotic-resistant biofilms. Small 8, 3016-3027 (2012).

18. Gu, H., Ho, P., Tsang, K. W. T., Wang, L. \& Xu, B. Using biofunctional magnetic nanoparticles to capture vancomycin-resistant enterococci and other Gram-positive bacteria at ultralow concentration. J. Am. Chem. Soc. 125, 15702-15703 (2003).

19. Ho, K.-C., Tsai, P.-J., Lin, Y.-S. \& Chen, Y.-C. Using biofunctionalized nanoparticles to probe pathogenic bacteria. Anal. Chem. 76, 7162-7168 (2004).

20. Gao, B. J. et al. Combining fluorescent probes and biofunctional magnetic nanoparticles for rapid detection of bacteria in human blood. Adv. Mater. 18, 3145-3148 (2006).

21. El-boubbou, K., Gruden, C. \& Huang, X. Magnetic Glyco-nanoparticles : A Unique Tool for Rapid Pathogen Detection, Decontamination, and Strain Differentiation. J. Am. Chem. Soc. 129, 13392-13393 (2007).

22. Kell, A. J. et al. Vancomycin-Modified Nanoparticles for Efficient Targeting and Preconcentration of Gram-Positive and Gram-Negative Bacteria. ACS Nano 2, 1777-1788 (2008).

23. Bean, C. P. \& Livingston, J. D. Superparamagnetism. J. Appl. Phys. 30, S120-S129 (1959).

24. Lu, Z. \& Yin, Y. Colloidal nanoparticle clusters: functional materials by design. Chem. Soc. Rev. 41, 6874-6887 (2012).

25. Liu, J. et al. Highly water-dispersible biocompatible magnetite particles with low cytotoxicity stabilized by citrate groups. Angew. Chemie - Int. Ed. 48, 5875-5879 (2009).

26. Mertz, W. The essential trace elements. Science 213, 1332-1338 (1981).

27. Ghuysen, J.-M. \& Hakenbeck, R. Bacterial cell wall. (Elsevier Science B.V., 1994).

28. Laloux, G. \& Jacobs-wagner, C. How do bacteria localize proteins to the cell pole? J. Cell Sci. 127, 11-19 (2014).

29. Lybarger, S. R. \& Maddock, J. R. Polarity in action: asymmetric protein localization in bacteria. J. Bacteriol. 183, 3261-3267 (2001).

30. Nicholson, W. L., Munakata, N., Horneck, G., Melosh, H. J. \& Setlow, P. Resistance of bacillus endospores to extreme terrestrial and extraterrestrial environments. Microbiol. Melecular Biol. Rev. 64, 548-572 (2000).

\section{Acknowledgements}

The authors thank Christoph M. Tang, Ziwei Deng, and Yansha Wu for useful discussions and assistance with the experiments. This work was supported by the EPSRC (Grant No. EP/J001902/1). X.Z. acknowledges financial support from the Young 1000 Talents Program.

\section{Author contributions}

B.P., D.G.A.L.A. and R.P.A.D. initialised the project. B.P. and X.Z. conceived the idea, and designed and performed the experiments, and analysed the data under the supervision of D.G.A.L.A. and R.P.A.D.. B.P. and R.P.A.D. wrote the paper with contributions from all co-authors. 


\section{Competing financial interests}

The authors declare no competing financial interests.

\section{Figure captions}

Figure 1 | Formation and characterisation of superparamagnetic nickel colloidal nanocrystal clusters (SNCNC). a, A schematic of the formation of SNCNC. b-d, Representative experimental transmission electron microscopy observations at $\mathbf{b}, 1 \mathrm{~min}, \mathbf{c}, 20 \mathrm{~min}$ and $\mathbf{d}, 1 \mathrm{~h}$, respectively. The inset in $\mathbf{b}$ shows a higher magnification image at $1 \mathrm{~min}$. e, Representative high-resolution transmission electron microscopy results revealing the cluster structure, the scale bars are $50 \mathrm{~nm}$ in e and $2 \mathrm{~nm}$ in the inset, respectively. f, and $\mathbf{g}$, energy dispersive X-ray spectroscopy and X-ray diffraction confirm the nickel nature of the clusters. $\mathbf{h}$, The temperature $(T)$ dependence of the mass magnetic moment $(M)$ as a function of external magnetic field $(H)$ and susceptibility $(\chi)$, and the inset shows the field-cooling (FC) and zero-field-cooling (ZFC) curves.

Figure 2 | Size and magnetisation manipulation of SNCNC. Representative scanning electron microscopy illustrates the tunability of the size by increasing the molar ratio between $\mathrm{NaOH}$ and $\mathrm{NiCl}_{2}$ from a, 5:2, b, 7.5:2, c, 12.5:2, to d, 17.5:2. e, Size distributions of the samples in a-d (for each histogram, $n>100$ particles were measured). f, Temperature-dependence of mass magnetic moment (M). Note that the $M$ of the SNCNC synthesised at $200{ }^{\circ} \mathrm{C}$ is shown in Figure $1 \mathrm{~h}$. The insets illustrate the recyclability of SNCNC in aqueous solution ( $\mathrm{SNCNC}$ are produced at $200{ }^{\circ} \mathrm{C}$ ). $\mathbf{g}$, The comparison of temperature impact on saturated $M$ and magnetisation per grain $(\mu)$, where $\mu_{B}$ is the Bohr magneton.

Figure 3 | Antibacterial activity and binding ability of the SNCNC. a, The binding of B. subtilis by SNCNC and superparamagnetic $\mathrm{Fe}_{3} \mathrm{O}_{4}$ colloidal nanocrystals (SFCN). The field strength of the neodymium magnets was $1.0-1.4 \mathrm{~T}$. b, Representative microscopic observation of SNCNC bound to B. subtilis. c, The antibacterial activity contrast between SNCNC and SFCN. d-f, Display the same experiments as in a-c but now for E. coli. The blue arrows point out the SNCNC attached to the bacteria. The scale bars are $20 \mu \mathrm{m}$ in $\mathbf{b}$ and $\mathbf{e}$, and $5 \mu \mathrm{m}$ in the insets.

Figure 4 | Quantitative analysis of the binding and antibacterial activity of SNCNC. The overall bacteria viability (representing the antibacterial activity) and unbound bacteria viability (reflecting the bacterial binding efficacy) as a function of time and SNCNC concentration; a, B. subtilis, b, E. coli, the reusability of SNCNC $(0.5 \mathrm{mg})$ for $\mathbf{c}, B$. subtilis: in each cycle the same SNCNC were mixed with approximately $10^{6}$ B. subtilis bacteria, and $\mathbf{d}$, bacterial spores from $B$. subtilis. Data are the means of three repeats, and error bars indicate standard deviations. 


\section{Methods}

The experimental details on the preparation and characterisation of the SNCNC, the controls and the bacteria experiments are described in the SI.

\section{Data availability}

The data that support the plots within this paper and other findings of this study are available from the corresponding authors upon reasonable request.

\section{Additional information}

Supplementary information is available in the online version of the paper. Reprints and permission information is available online at www.nature.com/reprints. Correspondence and requests for materials should be addressed to B.P. and R.P.A.D.. 

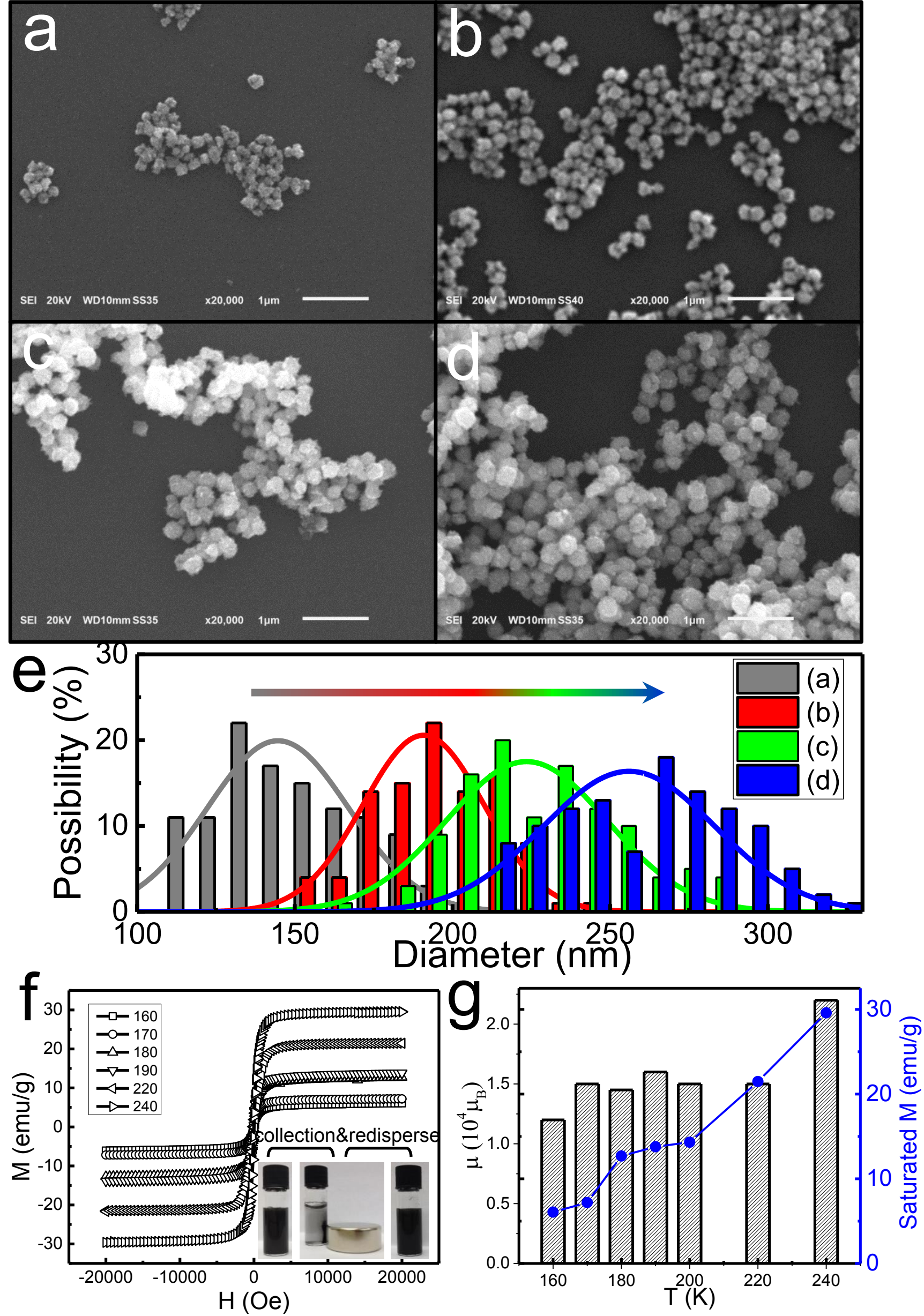


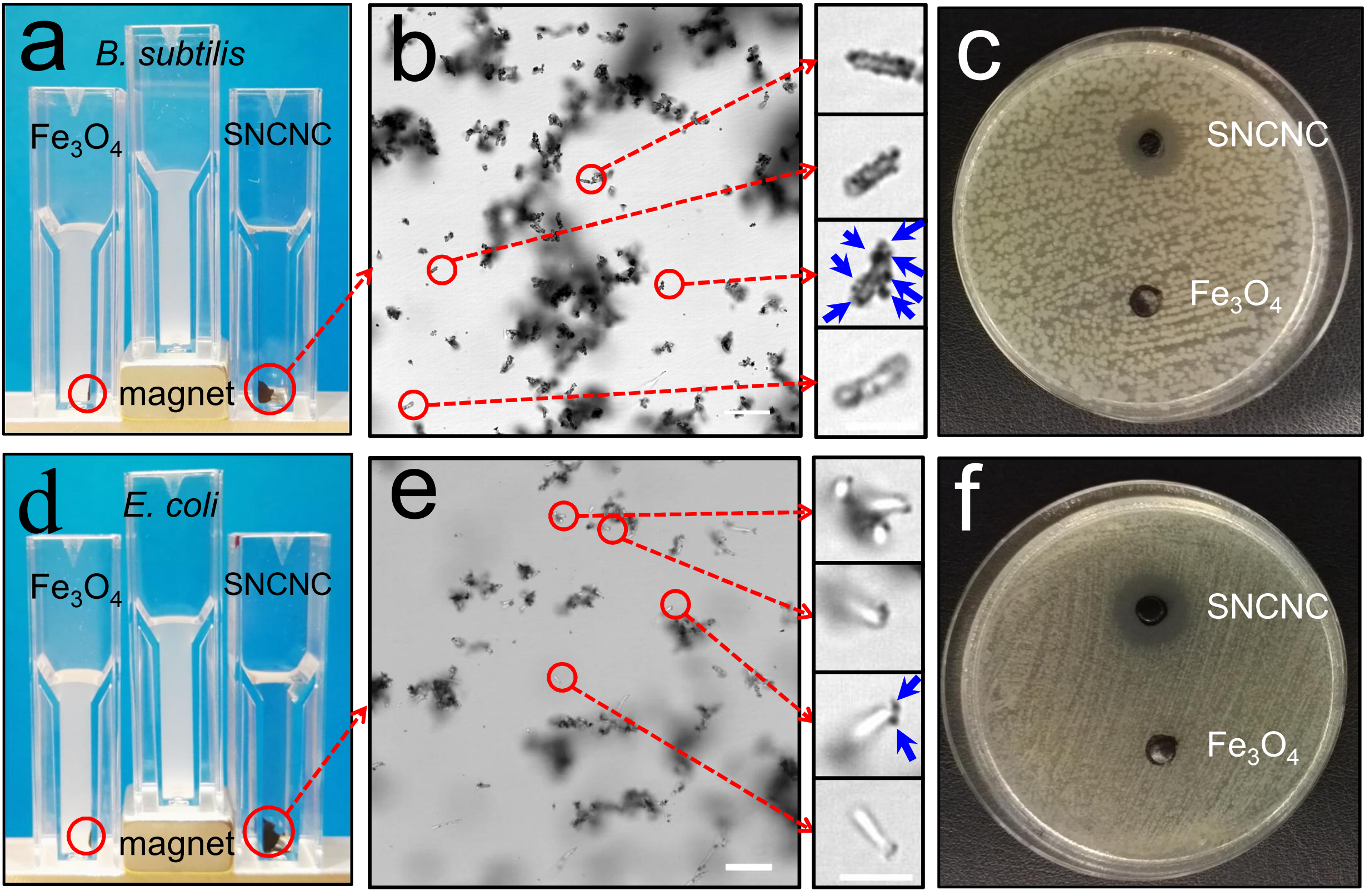



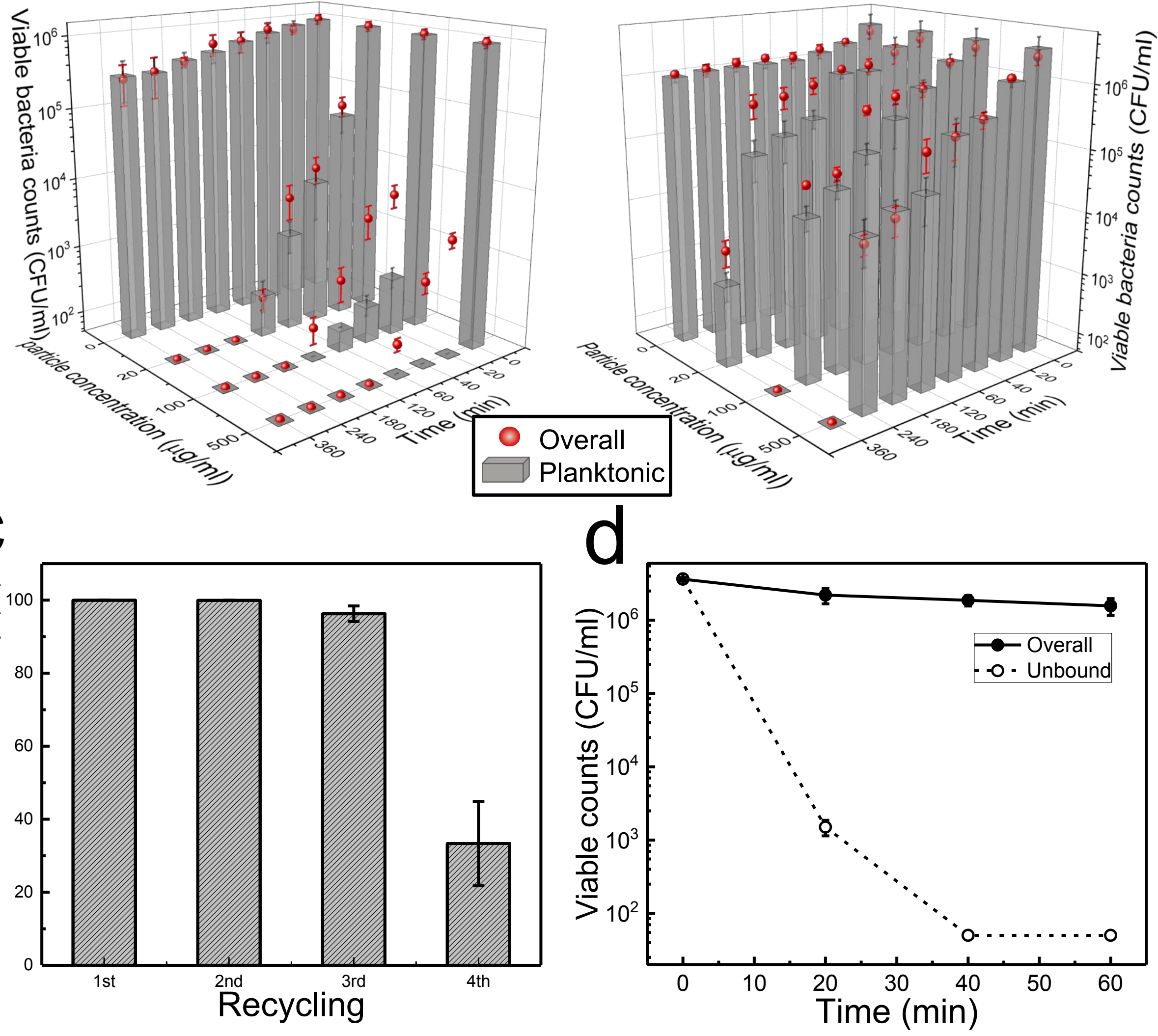